\title{
Modification of the standard model for the lanthanides
}

\author{
U. Lundin, I. Sandalov, O. Eriksson, and B. Johansson \\ Condensed Matter Theory group, Uppsala University, Box 530, SE-751 21 Uppsala, Sweden
}

(October 29, 2018)

\begin{abstract}
We show that incorporation of strong electron correlations into the Kohn-Sham scheme of band structure calculations leads to a modification of the standard model of the lanthanides and that this procedure removes the existing discrepancy between theory and experiment concerning the ground state properties. Within the picture suggested, part of the upper Hubbard $f$-band is occupied due to conduction band- $f$-mixing interaction (that is renormalized due to correlations) and this contributes to the cohesive energy of the crystal. The lower Hubbard band has zero width and describes fermionic excitations in the shell of localized $f$-s. Fully self-consistent calculations (with respect to both charge density and many-electron population numbers of the $f$-shell) of the equilibrium volume $V_{0}$ and the bulk modulus of selected lanthanides have been performed and a good agreement is obtained.
\end{abstract}

Published as: Sol. State Commun. 115, 7-12 (2000).

For strongly correlated systems, state-of-the-art band structure methods fail to give an accurate description. The reason for the failure is well-known: the strong intra-shell Hubbard repulsion, $U$, is underestimated in the calculations. If the $f$ - $f$ interaction is sufficiently strong, the $f$-shell forms a localized multiplet (Russel-Saunders coupling) and can accurately be treated as atomic like. This is the basic idea behind the so-called standard model of the lanthanides and many of the physical properties seem consistent with it, for example the Curie-Weiss behavior of the susceptibility $\mathrm{E}$, the gross features of the equilibrium volumes, and the structural properties 3 . When using ab initio calculations employing the local density approximation (LDA) and treating the $f$-electrons as core states, a too large equilibrium volume, $V_{0}$, is obtained (for all other elements, LDA underestimates the equilibrium volume). On the other hand, when the $4 f$ 's are treated as itinerant electrons, $V_{0}$ is much too low compared to experiment and the observed localized moment (Curie-Weiss law) is absent. Calculations by Delin et al 4 show that treating the $4 f$-electrons as core states is a very good approximation for the late lanthanides, but for the lighter lanthanides the disagreement between theory and experiment is gradually increasing when one goes from heavy to the lighter lanthanides. Thus, even though the standard model is essentially correct, it has to be modified slightly to better describe the cohesive properties of the lanthanides. Such a modification requires a method combining an ab initio band structure calculations (AIBSC) and a many-body approach. A variety of such methods are developef. The ideas of orbital polarization $\mathrm{E}, \mathrm{LDA}+\mathrm{U}$ method $\mathbf{6}$, Hartree-Fpck type of approximations, method of phase shift and more advanced methods, like three-body Faddeev equations 6 , have in some cases been successfully applied to systems with streng electron correlations (SEC) New approaches: on the basis of the Gutzwiller wave function 9 , dynamical mean field 10 and the LDA++-approach 11 have also been used for the description of different SEC systems. In many cases the AIBSC-based density of electron states is used as input to a model calculation using a non-self-consistent fixed lattice parameter and Hubbard $U$. However, since a large value of Hubbard repulsion restricts the available electron phase space, wave functions and, correspondingly, the matrix elements of mixing and hopping, changes. Recently an attempt to describe SEC by means of slave-boson method 12 (i.e. Hubbard $U=\infty$ ) build into the scheme of linear muffin-tin orbital method (LMTO) was made. The author found that the results are unsatisfactory 12 and had to use a scheme different from the standard slave-boson self-consistency schemes. However, in all these attempts the influence of SEC on the cohesive properties has not been questioned yet. Our approach to improve on the standard model is based on the following physical picture: the Hubbard repulsion is sufficiently large to form large energy gaps between the $(n-1)-, n$ - and $(n+1)$ electron configurations not only in the heavy but also in the light lanthanides. Near the atomic limit all $f$-electrons are in the strong coupling regime and hopping and mixing interactions lead to the formation of a lower Hubbard subbands (LHB, $|n-1\rangle \rightarrow|n\rangle$ transitions) and a upper sub-band (UHB, $|n\rangle \rightarrow|n+1\rangle$ transitions). In our case, however, the lower transitions are substantially below the bottom of the conduction bands (Ce is an exception) and, hence, they do not experience any mixing interaction. Hubbard $U$ increases with atomic number, due to the more localized nature of the $4 f$ 's, thus the LHB goes down in energy while the UHB goes up and therefore it experiences smaller mixing and is populated with fewer states. These many-electron local excitations correspond to localized core electrons within the one-electron picture. The upper transitions are in the energy region where the delocalized electrons do exist. Therefore, the overlap and mixing interaction may delocalize these transitions, forming an UHB, filled only by a small amount, $\eta$, of electrons. This picture corresponds to the multi-orbital Hubbard-Anderson model. The total occupation number can be written as $n_{f}=n+\eta$, where $n$ is an integer, which is determined by the nuclear charge valence, and $0<\eta<1$. Thus, most of the $f$-occupation is in the LHB. This is consistent with the observed Curie-Weiss behavior of the high-temperature (above $\mathrm{T}_{c}$ ) susceptibility. In the lanthanides the width of the appropriate $f$-sub band is much 
smaller than the Hubbard band gap. This allows one to formulate an-effective Schrödinger (or Dirac) equation (SE) for two different energy regions. As is known from Hubbard's models 13 , the single-electron spectral weight at $U=0$ is split by the Hubbard repulsion $U$ to a set of weights, corresponding to an electron transition from one ion state to another. These weights renormalize the matrix elements of the hopping and mixing interaction, which in turn, cause a narrowing of the bands due to correlations. The theory is developed for a non-orthogonal basis set, as for instance used in the linear muffin-tin orbital method in the atomic sphere approximation (LMTO-ASA) 44 . Here we apply the formalism only in the lowest approximation which requires less complicated changes for methods used in self consistent $a b$ initio calculation. Compared to conventional considerations of the periodical Anderson model (PAM) which do not include structure-dependent vertex corrections we solve the Schrödinger equation for the electrons in fields generated by the nuclei and electrons in every iteration, with the charge density obtained from a system of equations for Greens functions (GF) and spectral weights, thus renormalizing self-consistently the matrix elements of the PAM. On the other hand compared to the normal LDA approach we solve self-consistently additional system of equations for many-electron spectral weights and obtain a bandwidth which is reduced by correlations.

Technically our method, briefly described, consists of the following steps:

1. The total many-electron Hamiltonian is written in terms of an LMTO basis set.

2. The $f$-electron operators are transformed into a Hubbard representation.

3. Analytical calculations are performed for the-plectron Greens functions for the corresponding PAM in the LMTO representation in the Hubbard-I approximation 13 (strong-coupling regime).

4. Renormalizing factors to the $f$ - $(s p d)$-blocks of the LMTO Hamiltonian and overlap matrices are introduced which are derived from a comparison of the frequency and overlap matrixes. They arise due to strong correlations in the system for Greens functions.

5. Self-consistent, ab initio calculations of the system with SEC are performed.

In practice, the main effect of these steps consists of the separation 15 of the $f$-electron system into two subsystems and the correlation driven narrowing of the $f$-bands in the conventional LMTO Hamiltonian. This, as will be demonstrated below, leads to an improved description of the bonding of the lanthanide metals. In order to describe our theory in more detail, the secondary quantized full many-electron Hamiltonian, $\mathcal{H}$, is written in terms of an LMTO-basis set. An unperturbed Hamiltonian and a perturbation is formulated as $\mathcal{H}=\left(T+V_{n e}+V_{L D A}\right)+\left(H_{e e}-V_{L D A}\right)=\mathcal{H}_{0}+\mathcal{H}^{\prime}$. The main effect of the $f$-part single site correction from Coulomb interaction,

$$
\mathcal{H}_{U}^{\prime}=\frac{1}{2} \sum U_{m_{1} m_{2} m_{3} m_{4}} f_{m_{1} \sigma}^{\dagger} f_{m_{2} \sigma^{\prime}}^{\dagger} f_{m_{3} \sigma^{\prime}} f_{m_{4} \sigma}-\sum v_{l=3, \sigma}^{(L D A)} \sum f_{m_{l} \sigma}^{\dagger} f_{m_{l} \sigma}
$$

is to localize part of the $f$-spectra, similar to a so called LDA+U solution. Let us denote all non- $f$-operators in the LMTO representation as $c_{\mathbf{k} L}$. Then the LMTO part of the Hamiltonian, $\sum H_{L L^{\prime}}(\mathbf{k}) a_{\mathbf{k} L^{\dagger}}^{\dagger} a_{\mathbf{k} L^{\prime}}$, can be written as a sum of $s, p, d$, and $f$-electrons, and their mixing interaction,

$$
\begin{aligned}
& \mathcal{H}_{0}=\sum_{\mathbf{n} m_{l} \sigma} \epsilon_{f}^{0} f_{\mathbf{n} m_{l} \sigma}^{\dagger} f_{\mathbf{n} m_{l} \sigma}+\sum_{\mathbf{k} L, L^{\prime}} H_{L, L^{\prime}}(\mathbf{k}) c_{\mathbf{k} L}^{\dagger} c_{\mathbf{k} L^{\prime}}+ \\
& \sum_{\mathbf{n} \mathbf{m}, m_{l} \sigma m_{l}^{\prime}} t_{\mathbf{n} \mathbf{m}}^{m_{l} m_{l}^{\prime}} f_{\mathbf{n} m_{l} \sigma}^{\dagger} f_{\mathbf{m} m_{l}^{\prime} \sigma}+\sum_{\mathbf{k}, \mathbf{n}, L, m_{l} \sigma}\left[H_{L, m_{l} \sigma}(\mathbf{k}) e^{i \mathbf{k} \mathbf{R}_{\mathbf{n}}} c_{\mathbf{k} L \sigma}^{\dagger} f_{\mathbf{n} m_{l} \sigma}+\text { h.c. }\right],
\end{aligned}
$$

where $t_{\mathbf{n} m}^{m_{l} m_{l}^{\prime}}=\sum_{\mathbf{k}} e^{i \mathbf{k}\left(\mathbf{R}_{\mathbf{n}}-\mathbf{R}_{\mathbf{m}}\right)}\left[H_{m_{l} \sigma, m_{l}^{\prime} \sigma}(\mathbf{k})-\delta_{m_{l}, m_{l}^{\prime}} \epsilon_{f}^{0}\right]$ is the hopping matrix element between $f$-orbitals and $\epsilon_{f}^{0}=$ $\sum_{\mathbf{k}} H_{m_{l} \sigma, m_{l} \sigma}(\mathbf{k})$. Since the LMTO-ASA basis is non-orthogonal with an overlap matrix $\left\langle\chi_{\mathbf{n} L} \mid \chi_{\mathbf{m} L^{\prime}}\right\rangle=\mathcal{O}_{\mathbf{n} L, \mathbf{m} L^{\prime}}$, the anticommutator is $\left\{a_{\mathbf{n} L}, a_{\mathbf{m} L^{\prime}}^{\dagger}\right\}=\left(\mathcal{O}^{-1}\right)_{\mathbf{n} L, \mathbf{m} L^{\prime}}$. Therefore, the equation for the bare fermion Greens function, $G_{\mathbf{n} L, \mathbf{m} L^{\prime}}(\omega) \equiv-\left\langle T\left[a_{\mathbf{n} L}(t) a_{\mathbf{m} L^{\prime}}^{\dagger}(0)\right]\right\rangle_{\omega}$, for this Hamiltonian is

$$
\left[\omega \mathcal{O}_{\mathbf{n} L, \mathbf{m}_{1} L_{1}}-H_{\mathbf{n} L, \mathbf{m}_{1} L_{1}}\right] G_{\mathbf{m}_{1}, L_{1}, \mathbf{m} L^{\prime}}(\omega)=\delta_{\mathbf{n}, \mathbf{m}} \delta_{L, L^{\prime}} .
$$

Thus, we find (by construction) in the square brackets the conventional secular problem for the LMTO-ASA method (or any LDA based method). Introducing a full set of states in an orbital representation $\left.\left.\left.\mid \Gamma_{0}\right)=|0\rangle, \mid \Gamma_{1}\right)=|\gamma\rangle, \mid \Gamma_{2}\right)=$ $\left|\gamma, \gamma^{\prime}\right\rangle, \cdots\left(\gamma\right.$ denoting a single particle $f$-state, $\left.m_{l} \sigma\right)$ allows to express any single-site operator $\hat{\mathcal{A}}$ in terms of $X$ operators, $\hat{\mathcal{A}}=\sum_{\Gamma, \Gamma^{\prime}}\left\langle\Gamma|\hat{\mathcal{A}}| \Gamma^{\prime}\right\rangle X^{\Gamma, \Gamma^{\prime}}, X^{p q}=|p\rangle\langle q|$ and where $\left\langle X^{\Gamma, \Gamma}\right\rangle \equiv N_{\Gamma}$ is the occupation number for the state $\Gamma$. Now we can express the zero $f$-Greens functions, for the LMTO problem, in terms of $X$-Greens functions as $G_{\gamma}^{(0)}(\omega)=\sum_{a}\left|\left(f_{\gamma}\right)_{a}\right|^{2} P_{a} D_{a}(\omega)$, where $D_{a}(\omega)=\frac{1}{\omega-\Delta_{a}}$, and an $X$-operator GF $\mathcal{G}^{a \bar{a}}=P^{a} D_{a}$. Here $a=\left(\Gamma_{n}, \Gamma_{n+1}\right)$ $\left(\bar{a}=\left(\Gamma_{n+1}, \Gamma_{n}\right)\right)$ are possible Hubbard transitions, $P_{\Gamma_{n}, \Gamma_{n+1}}=N_{\Gamma_{n}}+N_{\Gamma_{n+1}}, \Delta_{\Gamma_{n+1}, \Gamma_{n}}=E_{\Gamma_{n+1}}-E_{\Gamma_{n}}-\mu, E_{\Gamma_{n}} \simeq$ $n \epsilon_{f}^{0}+U n(n-1) / 2$ and $\mu$ is the chemical potential.

The $f$-electron operators can be expanded in the Hamiltonian $\mathcal{H}$ into $X$-operators for the set of many-electron states described above. Then we arrive at an Anderson like model 


$$
\begin{array}{r}
\mathcal{H}=\sum_{\mathbf{n} \Gamma} E_{\Gamma} X_{\mathbf{n}}^{\Gamma \Gamma}+\sum_{\mathbf{k}, L, L^{\prime}} H_{L, L^{\prime}}(\mathbf{k}) c_{\mathbf{k} L}^{\dagger} c_{\mathbf{k} L^{\prime}}+ \\
\sum_{\mathbf{k}, \mathbf{n}, \mu, a}\left[H_{L, \mu}(\mathbf{k})\left(f_{\mu}\right)_{a} e^{i \mathbf{k} \mathbf{R}_{\mathbf{n}}} c_{\mathbf{k} L}^{\dagger} X_{\mathbf{n}}^{a}+h . c .\right] .+\sum_{\mathbf{n}, \mathbf{m}, a, b} t_{\mathbf{n} \mathbf{m}}^{a b} X_{\mathbf{n}}^{a} X_{\mathbf{m}}^{b} .
\end{array}
$$

Here $t_{\mathbf{n m}}^{a b}=\sum_{\mu, \nu}\left(f_{\mathbf{n} \mu}^{\dagger}\right)^{a}\left(f_{\mathbf{m} \nu}^{\dagger}\right)^{b} t_{\mathbf{n m}}^{\mu \nu}$. For the derivation of the equations for the Greens functions, $\mathcal{G}^{(c X)} \equiv$ $-\left\langle T\left[c_{\mathbf{n} L}(t) X_{\mathbf{n}}^{M \Gamma}(0)\right]\right\rangle$ and $\mathcal{G}^{(X X)} \equiv-\left\langle T\left[X_{\mathbf{n}}^{\Gamma M}(t) X_{\mathbf{n}}^{M \Gamma}(0)\right]\right\rangle$, (which will be compared to the equation for the regular Kohn-Sham (LMTO) problem) we need the anticommutators $\{c, X\}$, we find $\left\{c_{\mathbf{n} L}, X_{\mathbf{n}^{\prime}}^{\Gamma^{\prime} \Gamma}\right\}=$

$\sum_{\mathbf{n}_{1} \mu \Gamma_{1} \Gamma_{2}} \mathcal{O}_{\mathbf{n} L, \mathbf{n}_{1} \mu}^{-1}\left(f_{\mu}\right)_{\Gamma_{1}, \Gamma_{2}}\left\{X_{\mathbf{n}_{1}}^{\Gamma_{1}, \Gamma_{2}}, X_{\mathbf{n}^{\prime}}^{\Gamma^{\prime}, \Gamma}\right\}$. Physically, this implies that the $f$-part of the conduction-band wavefunction, coming from other sites, experiences strong correlations on the reference ion. The Hubbard-I as well as mean field approximation correspond to $\left\{X_{\mathbf{n}}^{\Gamma_{1}, \Gamma_{2}}, X_{\mathbf{n}}^{\Gamma^{\prime}, \Gamma}\right\} \simeq P_{\Gamma^{\prime}, \Gamma} \delta_{\Gamma^{\prime}, \Gamma_{2}} \delta_{\Gamma, \Gamma_{1}}$. Introducing renormalized "Fermi" operators $\tilde{f}^{\Gamma^{\prime}, \Gamma}=X^{\Gamma^{\prime}, \Gamma} / \sqrt{P_{\Gamma^{\prime}, \Gamma}}$. We see that the matrix form of the GF, $\mathcal{G}^{(i j)}$, with $i, j=c, X$, coincides with the GF of the Kohn-Sham equation, $G$, with the only difference being $A_{L, \mu} \rightarrow \tilde{A}_{L, \mu}=\sum_{\Gamma^{\prime}, \Gamma} A_{L, \mu}\left(f_{\mu}\right)_{\Gamma^{\prime}, \Gamma} \sqrt{P_{\Gamma^{\prime}, \Gamma}}$, where $A=H$ or $\mathcal{O}$. Thus the most essential, technical part of our theory leads to an effective secular equation which, when diagonalized gives eigenvalues with many-body corrections.

As an example let us consider praseodymium metal, which has two localized $f$-electrons. Therefore, $\left|\epsilon_{f}^{0}\right|$ has a value which gives an energy minimum for $n=2$. For briefness we will use the following notation for the $f$ orbitals: $\gamma=\left(m_{l}=3, \downarrow\right) \equiv 1,\left(m_{l}=2, \downarrow\right) \equiv 2$ and $\gamma=\nu$ for $\gamma \neq 1,2 . \quad \Gamma_{(12)} \equiv(12), \Gamma_{(12 \nu)} \equiv(12 \nu)$. Let us now consider a simple polarized solution, where the $f$-orbitals with $\gamma=1,2$ are fully occupied, while the rest of the $f$-electrons occupy the $f^{3}$-states, $\left|\Gamma_{3}\right\rangle=|1,2, \nu\rangle$. When mixing is absent, $n_{f}=2$ and $N_{(12)}^{0}=1$ whereas all other population numbers, $N_{\Gamma}^{0}$, are zero. Then $\mathcal{G}_{\gamma=1}^{0}=D_{2(12)}, \mathcal{G}_{\gamma=2}^{0}=D_{1(12)}$ and $\mathcal{G}_{\gamma=\nu}^{0}=D_{(12)(12 \nu)}$. We denote $\Delta_{(12) 2}=\Delta_{(12) 1} \equiv \Delta_{1}$, and $\Delta_{(12 \nu)(12)} \equiv \Delta_{2}$. In the limiting case, $\Delta_{2}, \Delta_{3} \rightarrow \infty$, no $f$ 's are present in the conduction band, and therefore this corresponds exactly to the limit of the standard model. The $f_{\nu}$-bands are slightly above the Fermi energy, and mixing interaction transfers $f$-character into the $s, p, d$-electron states. In a general case it is, of course, impossible to write equations for $N_{\Gamma}$ in terms of only orbital GF's, $\mathcal{G}_{\gamma}$, because equations for higher correlation functions are needed. However, if for simplicity we assume that these $12 \nu$-bands are occupied symmetrically, i.e., $N_{(12 \nu)}=(1 / 12) \sum_{\nu^{\prime}} N_{\left(12 \nu^{\prime}\right)}$, we immediately find

$$
\left\{\begin{array}{l}
n_{f}=2 \cdot N_{(12)}+3 \cdot \sum_{\nu} N_{(12 \nu)}=2+\eta \\
N_{(12)}+\sum_{\nu} N_{(12 \nu)}=1
\end{array}\right.
$$

i.e., $N_{(12 \nu)}=\eta / 12$ and $N_{(12)}=1-\eta$ (for the other rare-earth elements a slight modification of these expressions are needed). Thus, renormalization of the population numbers leads to the form of $f$-locators (single-site, $\mathbf{k}$-independent part of the GF)

$$
\mathcal{G}_{1}=\mathcal{G}_{2}=\frac{1-\eta}{\omega-\Delta_{1}}+\frac{\eta}{\omega-\Delta_{2}} \quad \text { and } \quad \mathcal{G}_{\nu}=\frac{1-11 \eta / 12}{\omega-\Delta_{2}}+\frac{11 \eta / 12}{\omega-\Delta_{3}}
$$

where $\Delta_{3} \equiv \Delta_{\left(12 \nu \nu^{\prime}\right)\left(12 \nu^{\prime}\right)}$. The center of the non-renormalized LDA- $f$-band is in $\omega=\Delta_{2}$ and $\mathcal{G}_{1}^{L D A}=\mathcal{G}_{2}^{L D A}=$ $\mathcal{G}_{\nu}^{L D A}=1 /\left(\omega-\Delta_{2}\right)$. Let us ignore for the moment the non-orthogonality. For a diagonal effective hopping $t_{\gamma}(\mathbf{k}, \omega)$, of any origin, we have $G_{\gamma}=\left[\left(G_{0, \gamma}^{(a t)}\right)^{-1}-t_{\gamma}\right]^{-1}$. Therefore, the expressions for the local, $f$-part, of the self-energies are $\Sigma_{\gamma}=\left(G_{\gamma}^{L D A}\right)^{-1}-U \sum_{\gamma^{\prime}}\left(1-\delta_{\gamma, \gamma^{\prime}}\right) n_{\gamma^{\prime}}-\left(G_{0, \gamma}^{(a t)}\right)^{-1}$. Since all poles in $\mathcal{G}_{\gamma}$ are well separated, one can formulate two effective Schrödinger equations near $\omega \sim \Delta_{1}$ and $\omega \sim \Delta_{2}$. Renormalization factors $\left(1-\left(\frac{\partial \Sigma}{\partial \omega}\right)_{\omega=\Delta_{i}}\right)$ are, of course, given by spectral weights (numerators) of the GF's in these poles. Since $\eta$ is small the bandwidths of the upper Hubbard sub bands for $\gamma=1,2$ are also small and, being above the Fermi level, they are empty. Within the scenario considered the lower sub bands have weight slightly less than one, $(1-\eta)$. For the bands $\nu, \Delta_{3}$ is far above the Fermi energy. Thus the upper sub bands are empty and the spectral weight in the pole $\omega=\Delta_{2}$ is $\left(1-\frac{11 \eta}{12}\right)$. Therefore, the effective Schrödinger equation for bands 1 and 2 at $\omega \sim \Delta_{1}=\Delta_{2}-U$ gives $\omega-\left(\Delta_{2}-U\right)-(1-\eta) t_{1}(\mathbf{k}, \omega)=0$, whereas for the bands $\nu$ at $\omega \sim \Delta_{2}$ we find $\omega-\Delta_{2}-(1-11 \eta / 12) t_{\nu}(\mathbf{k}, \omega)=0$.

Since in the case of LDA calculations with delocalized $f$-electrons the center of the $f$-band is $\sim \Delta_{2}$ we conclude that the potential for the 1st and 2 nd orbitals is shifted down by $U$ (similar to the LDA+U - result, 16 and self interaction correction (SIC)-theory 18 but without factors $n_{\mu}$ ). The next conclusion is that the described factors should be to introduced into the LMTO overlap and Hamiltonian matrices. Thus, we calculate the charge density and a self consistent potential, corresponding to the given $\eta$, where $\eta$ comes from the equation of self-consistency

$$
\eta=-\frac{1}{\pi} \sum_{\omega, \nu, \mathbf{k}} f(\omega) \operatorname{Im} \mathcal{G}_{\nu}^{(12,12 \nu),(12 \nu, 12)}(\omega+i \delta, \mathbf{k})
$$


since the parameter $P_{\Gamma^{\prime}, \Gamma}=1-\frac{11}{12} \eta$ renormalizes $\mathcal{H}$ and $\mathcal{O}$. Here $\mathcal{G}_{\nu}^{(12,12 \nu),(12 \nu, 12)}$ is obtained from the transformed secular matrix for the normal LMTO-GF's, $(\tilde{\mathcal{O}} E-\tilde{\mathcal{H}})^{-1}$, via $\tilde{f}$. For Pr we find the following picture. Two of the f-orbitals are located at an energy $-U$ lower than the remaining $f$-states and below the bottom of the conduction bands. Therefore, we treat those $f$-states as core states removing the somewhat arbitrary choice of a value for the Hubbard $U$. Compared to LDA+U and SIC methods, in our approach the spd-f hybridization is reduced by the renormalizing factor $\sqrt{P}$. The theory is generalized for the other lanthanides straightforwardly.

Before giving numerical examples of cohesive properties (e.g. equilibrium volume) obtained from our method we describe how the total energy was calculated. As is well-known, the equation for the exchange-correlation potential $v_{x c}$ derived by Sham 19 connects it with the exact self-energy of the electron system. Making use of a strong-coupling (SC) perturbation theory (PT) developed by us we have performed the analysis of the contributions to the self-energy and found that a there exist a one-to-one correspondence between the graphs for the self-energy in the standard weak-coupling (WC) PT (in e.g. the random phase approximation) and the sequence of graphs in SCPT. Using the facts that: a) Sham's equation 19 should produce a $v_{x c}$ corresponding to the particular SE choice; b) established correspondence between the WC and SC PT graphs; c) the statement of Kotani20, that the static random phase approximation reproduces well the DFT-LDA calculation with the standard choice of $v_{x c}$, we come to the conclusion that, within the approximation chosen, the original LDA functional form for $v_{x c}$ can be used. However, the charge density is calculated from renormalized fermions which differ from the standard fermions by the renormalizing factors $\sqrt{P}$. Hence the charge density, $\rho_{P}(r)$, also depends on $\sqrt{P}$. The mixing and hopping in the effective Kohn-Sham equation should be renormalized by the same $P$ as described above and a part of the renormalized $f$-electrons should be described as localized (lower transitions ) and the rest (coming from the upper transitions ) as delocalized. The separation is caused by self-interaction correction automatically generated in PT. Finally, the equation of selfconsistency for parameter $P$ (Eqn 7 ) should be added.

We have hence calculated the total energy using the regular LDA total energy expression, using the electron density $\rho_{P}(r)$, i.e. $E_{L D A}\left(\rho_{P}(r)\right.$, where the parameter $P$ which comes from electron correlations, is found self-consistently from additional equation and regulates distribution of spectral weight between lower and upper parts of energy. 

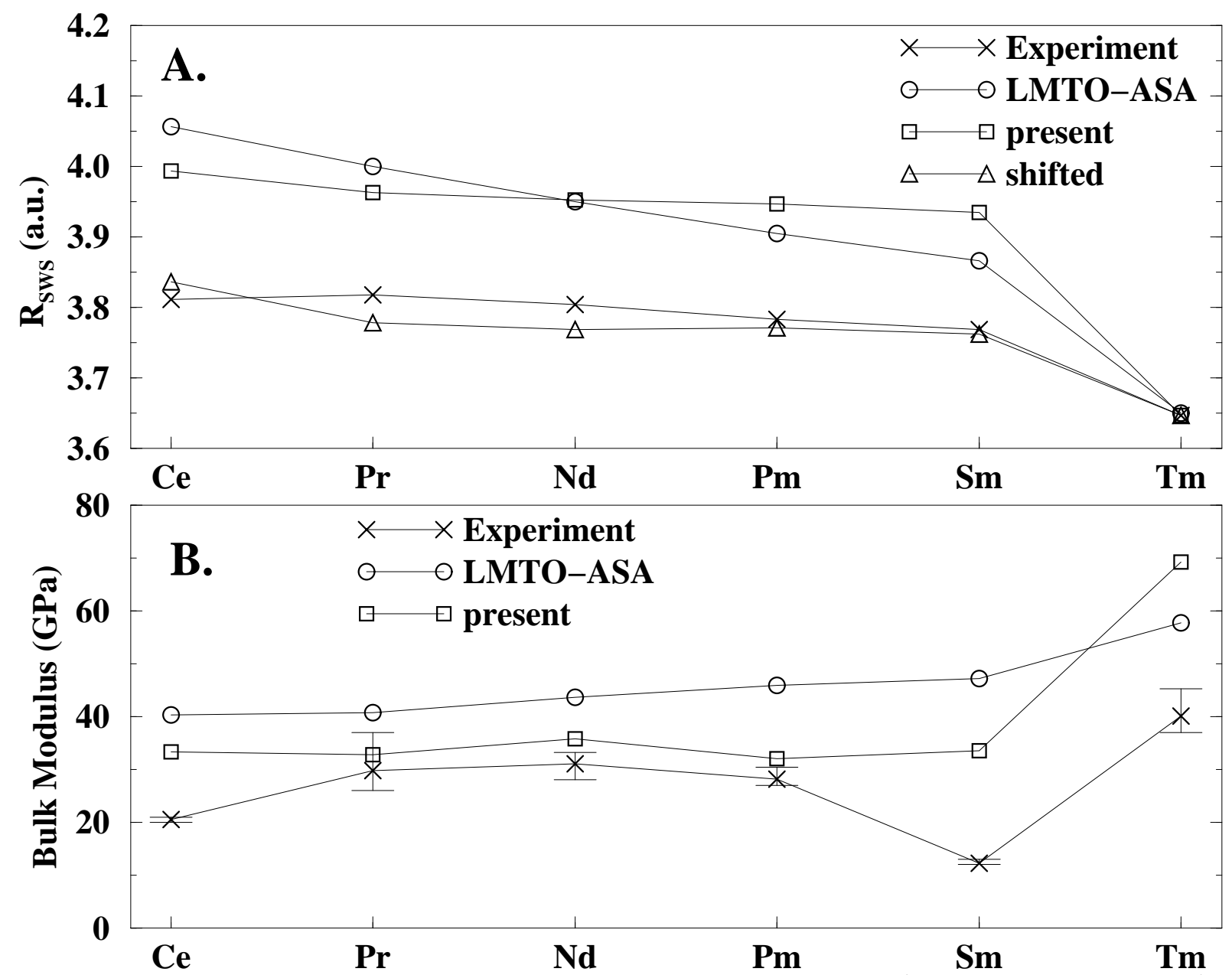

FIG. 1. Fig. A shows the equilibrium volumes, in experiment, LMTO-ASA (using the standard model), corrected LMTO-ASA and shifted theory, for some selected lanthanides. The basis-set shifts used are (in a.u.) for Ce $\Delta R_{s w s}=-0.157$, -0.185 for Pr, -0.184 for $\mathrm{Nd},-0.176$ for Pm, -0.172 for Sm and -0.003 for Tm. Fig. B shows the bulk modulus for the same elements. The slope of the lanthanide correction is reduced due to the contribution to the cohesive energy from the delocalized f's.

In Fig. 11A we show the equilibrium volumes of some selected lanthanides, from experiment, uncorrected Kohn-Sham calculations (LMTO-ASA) and from our corrected theory. Note that the many-body corrections are presented together with shifted data due to an incomplete basis set used in the LMTO-ASA calculations. This shift was calculated by comparing (LDA) volumes obtained from a full-potential-(FP)-LMTO, multi-basis set calculation with (LDA) volumes from LMTO-ASA calculations, $\Delta R_{s w s}=R_{0}^{F P}-R_{0}^{L M T O-A S A}$. An overall good agreement is obtained, and for the heavier lanthanides the renormalization correction becomes vanishingly small (as seen for Tm). The only parameter of our model, the position of the UHB with respect to the Fermi level, was taken from bremsstrahlung isochromat spectra (BIS)21. In principle this value can be found from a super cell calculation changing the $f$-occupancy at one site. For cerium, $\Delta_{n, n+1}$ is $\simeq 3.5 \mathrm{eV}$ above the Fermi level, and shifting this to $3.5 \pm 1 \mathrm{eV}$, only affected the equilibrium volume with $\pm 0.5 \%$. For samarium the position of the $\mathrm{UHB}$ is $\simeq 0.6 \mathrm{eV}$, and changes in the position with $\pm 0.1 \mathrm{eV}$, changed the volume with $\pm 0.8 \%$. For the other elements changes were of the same order. Therefore, we conclude that the results are rather insensitive with respect to the position of the UHB. Fig. 11B shows the bulk modulus. Note that this value is quite sensitive to the fitting procedure (pressure-volume fit) and to the structure, thus, it should be considered only as an approximate value.

The physical picture obtained is consistent with previous theoretical work on Fermi surfaces 17 where a better agreement between theory and experiment was obtained when including, ad-hoc, the 4f states as valence electrons even though part of these $4 \mathrm{f}$ states were treated as occupied gore states. It is also consistent with results obtained from self interaction corrected density functionals on $\operatorname{Pr}$ metal18. Unlike the method of orbital polarization the present theory is a true many-body correction, and thus presents a different physical approach. The decisive parameter in our case (unlike to the $\mathrm{LDA}+\mathrm{U}$ method) is not the absolute value of the Hubbard $U$ but the position of the transition 
$E\left(\Gamma_{n+1}\right)-E\left(\Gamma_{n}\right)$ with respect to Fermi energy. We have taken this parameter from experimental datan and this led us to an improvementin the calculated cohesive properties. First loop corrections, affecting the position of the levels, are small in our case22. Fluctuations coming from charge excitations are expected to alter the situation at non-zero temperature, however, this remains an open question.

We have shown that many-body corrections to the standard model improves the description of the cohesive properties of the lanthanides, and explains the observed discrepancy for the equilibrium volumes for the light lanthanides. The only input to the present theory is the position of the upper Hubbard transition, $\Delta_{\Gamma+1, \Gamma}$, which was taken from bremsstrahlung isochromat spectra (BIS) data. We found that a moderate change in energy of this transition gives only a minor change in the equilibrium volume. The calculated volumes are in good agreement with the experimental ones, which means that the excited states contributes to the ground state properties. The presented theory is designed to deal with strongly correlated electron systems and is integrated in an electronic structure method, and made fully self-consistent. It describes an additional contribution to the chemical bonding from a fraction $f$-electrons hybridized with the conduction electrons. Compared to for instance the $\mathrm{LDA}+\mathrm{U}$, orbital polarization or SIC methods the present theory involves a decrease of mixing due to strong correlations, causing the $f$-bandwidth to be reduced.

We are thankful for financial support from the Swedish Natural Science Research Council. The critical reading of our manuscript by Prof. Helmut Eschrig is greatly appreciated.

${ }^{1}$ G. H. Lander and G. R. Choppin, Handbook on the Physics and Chemistry of the Rare Earths (North-Holland, Amsterdam, 1993).

${ }^{2}$ B. I. Min, H. J. F. Jansen, T. Oguchi and A. J. Freeman, J. Magn. Magn. Matter 61 (1986) 139; O. Eriksson, B. Johansson and M. S. S. Brooks J. Less-Common Met. 158 (1990) 207.

${ }^{3}$ J. C. Duthie and D. G. Pettifor, Phys. Rev. Lett. 38 (1977) 564.

${ }^{4}$ A. Delin, L. Fast, O. Eriksson and B. Johansson, Phys. Rev. B 58 (1998) 4345.

${ }^{5}$ O. Eriksson, B. Johansson and M. S. S. Brooks, Phys. Rev. B 41 (1990) 7311.

${ }^{6}$ B.Brandow, Adv. Phys. 26, 651 (1977); A.M.Boring, R.C.Albers, O.Eriksson, and D.D.Koelling, Phys. Rev. Lett. 68, 2652 (1992); V. Anisimov, J. Zaanen and O. K. Andersen,Phys. Rev. B 44 (1991) 943.

${ }^{7}$ P. Fulde Electron Correlations in Molecules and Solids (Springer-Verlag, Berlin, 1991); G. Zwicknagl, Adv. Phys. 41 (1992) 203.

${ }^{8}$ J. Igarashi, P. Unger, K. Hirai and P. Fulde,Phys. Rev. B 49 (1994) 16181; F. Manghi, C. Calandra and S. Ossicini, Phys. Rev. Lett. 73 (1994) 3129; F. Manghi, V. Bellini and C. Arcangeli, Phys. Rev. B 56 (1997) 7149.

${ }^{9}$ N. E. Zein, Phys. Rev. B $\mathbf{5 2}$ (1995) 11813.

10 A. Georges, G. Kotliar, W. Krauth and M. J. Rozenberg, Rev. Mod. Phys. 68 (1996) 13.

11 A. I. Lichtenstein and M. I. Katsnelson, Phys. Rev. B B57 (1998) 6884.

12 M. Biagini, Phys. Rev. Lett. B77 (1996) 4066.

13 J. Hubbard, Proc. Roy. Soc. 276A (1963) 238; J. Hubbard, Proc. Roy. Soc. 277A (1964) 237; I. S. Sandalov and F. I. Filatiev, Physica B 162 (1990) 139; I. S. Sandalov, Proc. Int. Conf. on Physics of Transition Metals (Darmstadt) (World Scientific, 1992) 62.

${ }^{14}$ O. K. Andersen, Phys. Rev. B 12 (1975) 3060; H. L. Skriver, The LMTO Method (Springer, Berlin 1984).

${ }^{15}$ E. V. Kuz'min and I. S. Sandalov, Zh. Eksp. teor. fiz. 68 (1975) 1388.

${ }^{16}$ A. M. Boring, R. C. Albers, O. E. Eriksson and D. D. Koelling, Phys. Rev. Lett. 68 (1992) 2652.

${ }^{17}$ M. R. Norman and D. D. Koelling, Handbook on the Physics and Chemistry of the Rare Earths 17 (North-Holland, Amsterdam, 1993) 1.

${ }^{18}$ W. M. Temmerman, Z. Szotek and H. Winter, Phys. Rev. B 47 (1993) 1184; A. Svane, J. Trygg, B. Johansson and O. Eriksson, Phys. Rev. B 56 (1997) 7143.

${ }^{19}$ L. J. Sham Phys. Rev. B 32 (1985) 3876.

${ }^{20}$ T. Kotani J. of Phys. Condensed Matter 10 (1998) 9241.

${ }^{21}$ Y. Baer and W. D. Schneider, Handbook on the Physics and Chemistry of the Rare Earths 10 (North-Holland, Amsterdam, 1987) 1.

${ }^{22}$ U. Lundin, O. Eriksson, B. Johansson and I. S. Sandalov, 1997 Physica B 230-232 (1997) 445. 